\title{
Supplemental Information for Publication: Time-domain Line-shape Analysis from 2D Spectroscopy to Precisely Determine Hamiltonian Parameters for a Photosynthetic Complex
}

Brian S. Rolczynski, ${ }^{1}$ Shu-Hao Yeh, ${ }^{1,2}$ Polina Navotnaya, ${ }^{1}$ Lawson T. Lloyd, ${ }^{1}$ Alan R. Ginzburg, ${ }^{1}$ Haibin Zheng, ${ }^{1}$ Marco A. Allodi, ${ }^{1}$ John P. Otto, ${ }^{1}$ Khuram Ashraf, ${ }^{3, \dagger}$ Alastair T. Gardiner, ${ }^{3, \#}$ Richard J. Cogdell, ${ }^{3}$ Sabre Kais, ${ }^{2,4}$ and Gregory S. Engel ${ }^{1, *}$

${ }^{1}$ Department of Chemistry, The Institute for Biophysical Dynamics, and The James Franck Institute, The University of Chicago, Chicago, Illinois 60637, USA

${ }^{2}$ Qatar Environment and Energy Research Institute, Qatar Foundation, Doha, Qatar

3Institute of Biomedical and Life Sciences, Glasgow Biomedical Research Centre, University of Glasgow, Glasgow G12 8QQ, Scotland, United Kingdom

${ }^{4}$ Department of Chemistry, Purdue University, West Lafayette, Indiana, USA

$\dagger$ Current affiliation: Department of Biochemistry, Physiology and Molecular Biophysics, Columbia University, New York, New York 10027

\# Current affiliation: Laboratory of Anoxygenic Phototrophs, Centre ALGATECH, Novohradska 237, 37901 Třeboň, Czech Republic

* Corresponding author, email: gsengel@uchicago.edu 


\section{Methods}

2D electronic spectroscopy. A Legend Elite regenerative amplifier is seeded by a Micra oscillator (Coherent, Inc.) to produce a pulsed beam with a $5 \mathrm{kHz}$ repetition rate. A whitelight spectrum is produced by focusing the beam into argon gas. A MIIPS pulse shaper (Biophotonic Solutions, Inc.) is used to compress the pulse to $14 \mathrm{fs}$ and shape its spectrum to an approximate Gaussian line shape (fig. S1). It is subsequently used in a 2D electronic spectrometer, which was described previously. ${ }^{1}$ We perform 2D spectroscopy by illuminating the sample with four beams in a boxcar geometry. By sampling the signal in this phase matched direction, we ensure that beam 1 contributes a negative wavevector while beams 2 and 3 contribute positive ones. Therefore, measuring the portion of the signal propagating in the direction of the local oscillator (beam 4) yields either the rephasing $\left(\mathrm{ks}_{\mathrm{s}}=\right.$ $\left.-\mathrm{k}_{1}+\mathrm{k}_{2}+\mathrm{k}_{3}\right)$ or non-rephasing $\left(\mathrm{k}_{\mathrm{s}}=\mathrm{k}_{2}-\mathrm{k}_{1}+\mathrm{k}_{3}\right)$ signals, depending on whether beams 1 or 2 arrive at the sample first. As a result, aside from pulse mis-ordering near $\tau=0$ due to the non-zero pulse widths, the rephasing signal occurs at $\tau>0$ and the non-rephasing signal occurs at $\tau<0$. The time delays for pulses 1 and 2 have a range of several picoseconds, allowing long-range scans to be performed in coherence time. ${ }^{1}$ This long range is necessary to perform the Lorentz-Gauss filtering methods effectively, as discussed in the main text.

Hierarchical equations of motion. To describe the influence of environmental fluctuations (due to protein, solvent, or intramolecular interactions) on excitonic energy transfer, a non-perturbative method called the hierarchical equations of motion (HEOM) has been implemented. HEOM was first derived by Tanimura and Kubo, ${ }^{2}$ employing the Feynman-Vernon double path integrals. ${ }^{3}$ 
We assume that each site is coupled to a phonon bath independently. Each phonon bath is modeled as a series of overdamped harmonic oscillators, and therefore the Drude spectral density $J(\omega)=2 \lambda \gamma \omega / \pi\left(\omega^{2}+\gamma^{2}\right)$ was implemented. Adopting the parameters commonly used in other HEOM studies of FMO, ${ }^{4}$ the Drude decay constant $\gamma$ and reorganization energy $\lambda$ are set to $0.01 \mathrm{fs}^{-1}$ and $35 \mathrm{~cm}^{-1}$, respectively. Under this Drude spectral density, the correlation function can be expanded as a sum of exponential functions: $C(t)=\sum_{k=0}^{\infty} c_{k} e^{-v_{k} t}$, where $v_{0}=\gamma$ and Matsubara frequencies $v_{k}=2 \pi k / \beta \hbar$. The coefficients $c_{k}$ are given by equations $\mathrm{S} 1$ and $\mathrm{S} 2$.

$c_{0}=\hbar \lambda \gamma\left[\cot \left(\frac{\beta \hbar \gamma}{2}\right)-i\right]$,

$c_{k}=\frac{4 \lambda \gamma}{\beta} \frac{v_{k}}{v_{k}^{2}-\gamma^{2}}$, for $k \geq 1$

We implemented a modified version of HEOM (named scaled HEOM) proposed by Shi et al., ${ }^{5}$ which improves the numerical convergence with respect to the number of Matsubara frequencies included in the correlation function. The scaled HEOM is shown in equation S3 after the Ishizaki-Tanimura truncation scheme $v_{k} e^{-v_{k} t} \simeq \delta(t)$ has been applied to the $k>$ $K$ terms in the correlation function $C(t)$.

$$
\begin{aligned}
& \frac{d}{d t} \tilde{\rho}_{\boldsymbol{n}}=-\frac{i}{\hbar}\left[\widehat{H}_{S}, \tilde{\rho}_{\boldsymbol{n}}\right]-\left(\sum_{j=1}^{N} \sum_{k=0}^{K} n_{j k} v_{k}\right) \tilde{\rho}_{\boldsymbol{n}}-\frac{1}{\hbar^{2}} \sum_{k=K+1}^{\infty}\left(\frac{c_{k}}{v_{k}}\right) \sum_{j=1}^{N}\left[\widehat{V}_{j},\left[\widehat{V}_{j}, \tilde{\rho}_{\boldsymbol{n}}\right]\right]- \\
& \frac{i}{\hbar} \sum_{j=1}^{N} \sum_{k=0}^{K} \sqrt{\left(n_{j k}+1\right)\left|c_{k}\right|}\left[\widehat{V}_{j}, \tilde{\rho}_{\boldsymbol{n}_{j k}^{+}}\right]-\frac{i}{\hbar} \sum_{j=1}^{N} \sum_{k=0}^{K} \sqrt{\frac{n_{j k}}{\left|c_{k}\right|}}\left(c_{k} \widehat{V}_{j} \tilde{\rho}_{\boldsymbol{n}_{j k}}-c_{k}^{*} \tilde{\rho}_{\boldsymbol{n}_{j k}^{-}} \hat{V}_{j}\right)
\end{aligned}
$$

$\widehat{H}_{S}$ is the system Hamiltonian which has the form of $\left[\begin{array}{ccc}0 & 0 & 0 \\ 0 & H_{1 e x} & 0 \\ 0 & 0 & H_{2 e x}\end{array}\right]$. Here $H_{1 e x}=$ $\sum_{j=1}^{N}\left[\left(E_{j}+\lambda\right)|j\rangle\langle j|+\sum_{k \neq j} J_{j k}(|j\rangle\langle k|+| k\rangle\langle j|)\right] \quad$ and $\quad\left\langle j k\left|H_{2 e x}\right| j^{\prime} k^{\prime}\right\rangle=\delta_{j j^{\prime}} \delta_{k k^{\prime}}\left(E_{j}+E_{k}\right)+$ 
$\delta_{j j^{\prime}}\left(1-\delta_{k k^{\prime}}\right) J_{k k^{\prime}}+\delta_{j k^{\prime}}\left(1-\delta_{k j^{\prime}}\right) J_{k j^{\prime}}+\delta_{k j^{\prime}}\left(1-\delta_{j k^{\prime}}\right) J_{j k^{\prime}}+\delta_{k k^{\prime}}\left(1-\delta_{j j^{\prime}}\right) J_{j j^{\prime}} \quad$ are $\quad$ the single-exciton and bi-exciton Hamiltonian respectively, where $E_{j}$ is the site energy for the $j$ th pigment and $J_{j k}$ is the inter-site coupling between the $j$ - and $k$-th pigment. In Eq. S3, $j$ represents the site index, $V_{j}$ is the excitonic projection operator, and $\boldsymbol{n} \equiv$ $\left\{\left\{n_{10}, n_{11}, \cdots, n_{1 \mathrm{~K}}\right\}, \cdots,\left\{n_{N 0}, n_{N 1}, \cdots, n_{N K}\right\}\right\}$ is a vector of indices. The density matrices $\tilde{\rho}_{\boldsymbol{n}}$ are coupled to the ones with indices $\boldsymbol{n}_{j k}^{+}=\left\{\left\{n_{10}, n_{11}, \cdots, n_{1 K}\right\}, \cdots,\left\{n_{j 0}, \cdots, n_{j k} \pm\right.\right.$ $\left.\left.1, \cdots, n_{j K}\right\}, \cdots,\left\{n_{N 0}, n_{N 1}, \cdots, n_{N K}\right\}\right\}$. Each density matrix $\tilde{\rho}_{\boldsymbol{n}}$ has a hierarchy tier of $L$, which is defined by $L \equiv \sum_{j, k} n_{j k}$, and for simulating the 2D spectra of FMO and homodimer we included hierarchy matrices till $L_{\max }=2$ and $L_{\max }=6$, respectively.

The simulation of 2D electronic spectra starts with the calculation of third-order optical response function (equation S4).

$R^{(3)}(t, T, \tau)=-\frac{i}{\hbar^{3}}\langle[[[\hat{\mu}(\tau+T+t), \hat{\mu}(\tau+T)], \hat{\mu}(\tau)], \hat{\mu}]\rangle$,

Here, $\langle\cdots\rangle \equiv \operatorname{Tr}\left[\cdots \hat{\rho}_{\text {eq }}\right] \quad . \quad \hat{\mu}=\sum_{j=1}^{N} \mu_{j}|0\rangle\left\langle j\left|+\sum_{j=1}^{N-1} \sum_{k=j+1}^{N} \mu_{j}\right| k\right\rangle\left\langle j k\left|+\mu_{k}\right| j\right\rangle\langle j k| \quad$ is the transition dipole operator, and within the Heisenberg picture it has the form of $\hat{\mu}(t) \equiv$ $e^{i \widehat{H} t / \hbar} \hat{\mu} e^{-i \widehat{H} t / \hbar}$. After applying the rotating wave approximation, the total response of the 2D spectral signal can be obtained by the non-rephasing and the rephasing contributions. The non-rephasing part is calculated by equation $\mathrm{S} 5$.

$R_{\mathrm{NR}}^{(3)}(t, T, \tau)=-\frac{i}{\hbar^{3}} \operatorname{Tr}\left[\hat{\mu}_{\hat{G}(t)} \widehat{\mu}_{+}^{\times} \hat{\mathcal{G}}(T) \widehat{\mu}_{+}^{\times} \hat{G}(\tau) \widehat{\mu}_{-} \widehat{\rho}_{e q}\right]$,

and the rephasing part is calculated by equation $\mathrm{S} 6$. 


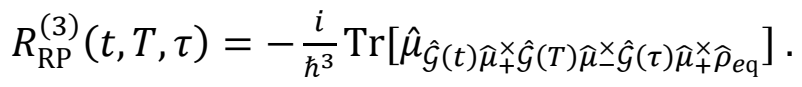

After performing a 2D Fourier transform with respect to the $\tau$ and $t$ times, the 2D signal are obtained as equations S7 and S8.

$S_{\mathrm{NR}}^{(3)}\left(\omega_{3}, T, \omega_{1}\right)=\operatorname{Im} \int_{0}^{\infty} d \tau \int_{0}^{\infty} d t_{3} e^{i\left(\omega_{1} \tau+\omega_{3} t\right)} R_{\mathrm{NR}}^{(3)}(t, T, \tau),(\mathrm{S} 7)$

$S_{\mathrm{RP}}^{(3)}\left(\omega_{3}, T, \omega_{1}\right)=\operatorname{Im} \int_{0}^{\infty} d \tau \int_{0}^{\infty} d t_{3} e^{i\left(-\omega_{1} \tau+\omega_{3} t\right)} R_{\mathrm{RP}}^{(3)}(t, T, \tau) \cdot$ (S8)

The combined signal used in the current study is defined as $S_{\mathrm{C}}^{(3)}\left(\omega_{3}, T, \omega_{1}\right)=$ $\operatorname{Abs}\left(\int_{0}^{\infty} d \tau \int_{0}^{\infty} d t_{3} e^{i\left(\omega_{1} \tau+\omega_{3} t\right)} R_{\mathrm{NR}}^{(3)}(t, T, \tau)+\int_{0}^{\infty} d \tau \int_{0}^{\infty} d t_{3} e^{i\left(-\omega_{1} \tau+\omega_{3} t\right)} R_{\mathrm{RP}}^{(3)}(t, T, \tau)\right)$.

A reorganization energy of $35 \mathrm{~cm}^{-1}$ was used for all chromophores, with a bath relaxation time of $100 \mathrm{fs}$. For more theoretical detail on simulating 2D electronic spectra with HEOM, see the following references..$^{4,6}$

\section{Spectra of the sample and light}

The laser spectrum is shaped to an approximate Gaussian peak using a MIIPS (Biophotonic Solutions, Inc.). The absorption spectrum of the Fenna-Matthews-Olson complex (FMO) overlaps with the light spectrum (fig. S1). 


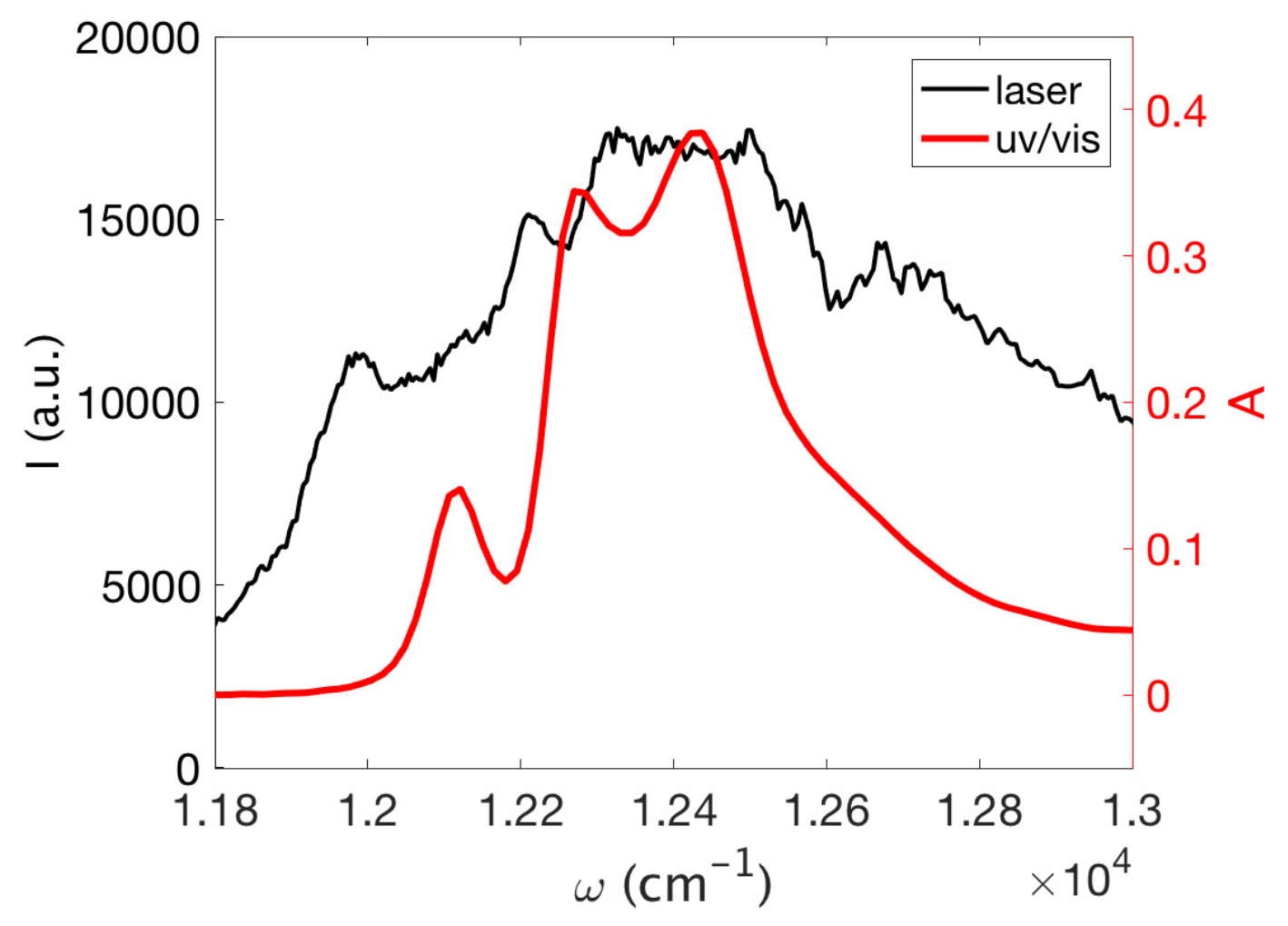

Figure S1. The laser spectrum (black) and FMO measured linear absorption spectrum (red) overlap significantly. 


\section{The application of Lorentz-Gauss filters, using calculated spectra}

Lorentz-Gauss filters were applied to the experimental and calculated 2D spectra along both the coherence- and rephasing-time dimensions. They were first tested with calculated spectra of a model homodimer system and a calculated FMO spectrum, before finally being used on the experimental FMO spectrum.

Unlike experimental spectra, an advantage of calculated spectra is that their Hamiltonian is known exactly. Therefore, the expected peak energies within the spectrum are known and can be compared to the obtained peaks in the filtered data. The homodimer system was composed of two identical chromophores, where each chromophore is modeled as an electronic two-level system with a transition energy of $12000 \mathrm{~cm}^{-1}$. The couplings were set to 100 or $25 \mathrm{~cm}^{-1}$. In these cases, the expected peak positions are 11900 and $12100 \mathrm{~cm}^{-1}$, or 11975 and $12025 \mathrm{~cm}^{-1}$, respectively. The dihedral angle between the two transition dipole vectors was set to $2 \pi / 5$. The parameters used in HEOM for spectral calculations are the same as the ones used in FMO. Figure S2 shows representative examples with 100 and $25 \mathrm{~cm}^{-1}$ of coupling. These spectra indicate, using a basic system, that the filtering method is able to reveal cross peaks even with only small coupling. 

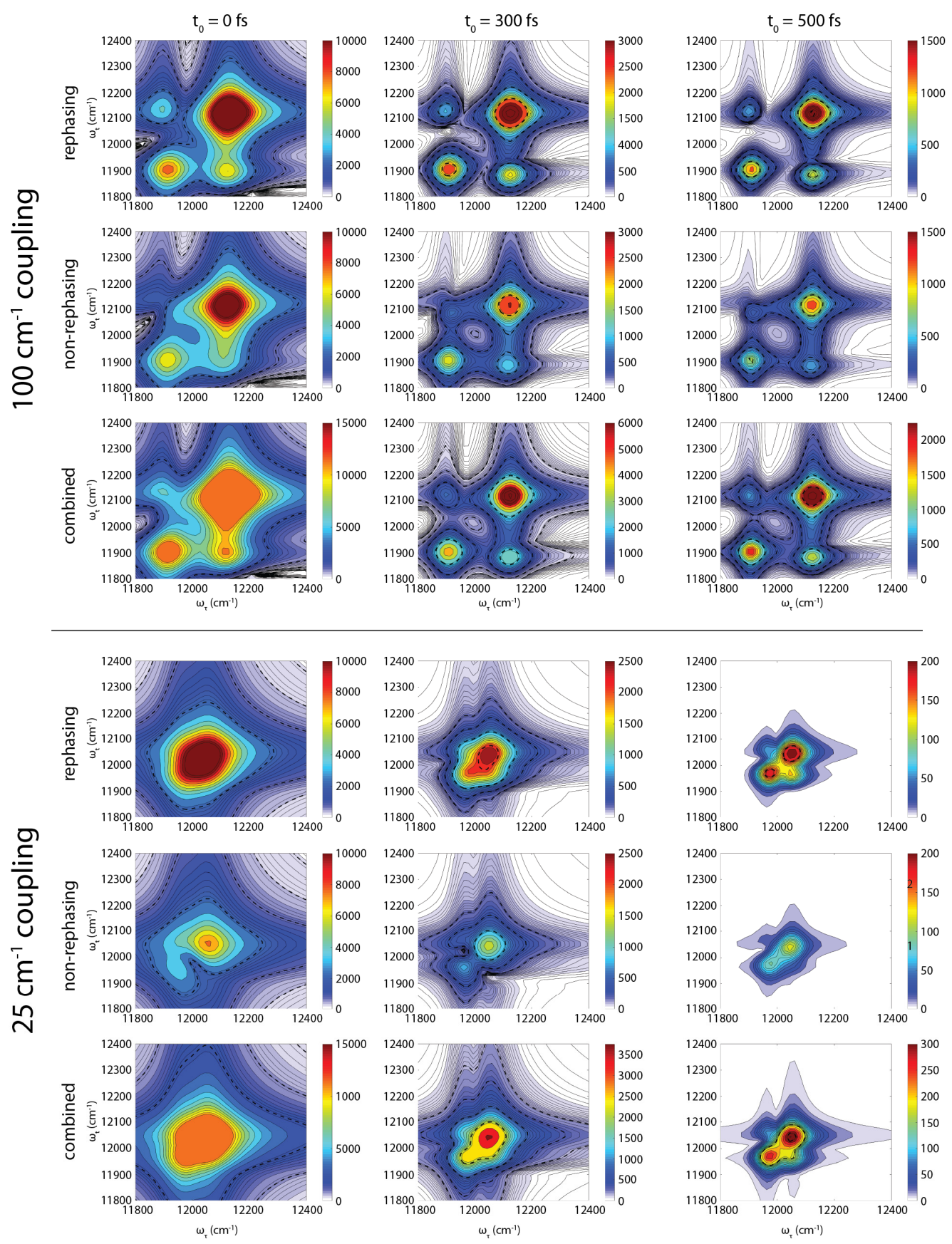

Figure S2. Two-dimensional spectra are shown, obtained from calculation of a model homodimer system. The rephasing, non-rephasing, and combined spectra are shown, using Lorentz-Gauss filters with $t_{0}^{\prime}=0,300$, and $500 \mathrm{fs}$. The Hamiltonian was constructed using $12000 \mathrm{~cm}^{-1}$ site wavenumbers and either 100 or $25 \mathrm{~cm}^{-1}$ coupling. Therefore, the expected peak positions are 11900 and $12100 \mathrm{~cm}^{-1}$, or 12975 and $12025 \mathrm{~cm}^{-1}$, respectively. The spectra contain peaks at these expected positions. 


\section{The application of Lorentz-Gauss filters on experimental data}

The Lorentz-Gauss filters are applied in the $(\tau, \mathrm{T}, t)$ domain. A representative example is shown in fig. S3. The Lorentz-Gauss filters are applied along both the $\tau$ and $t$ domains, and the resulting complex-valued data set is Fourier transformed in both of these axes to obtain the filtered 2D spectra. To observe the effect of increasing $t_{0}^{\prime}$, an example is
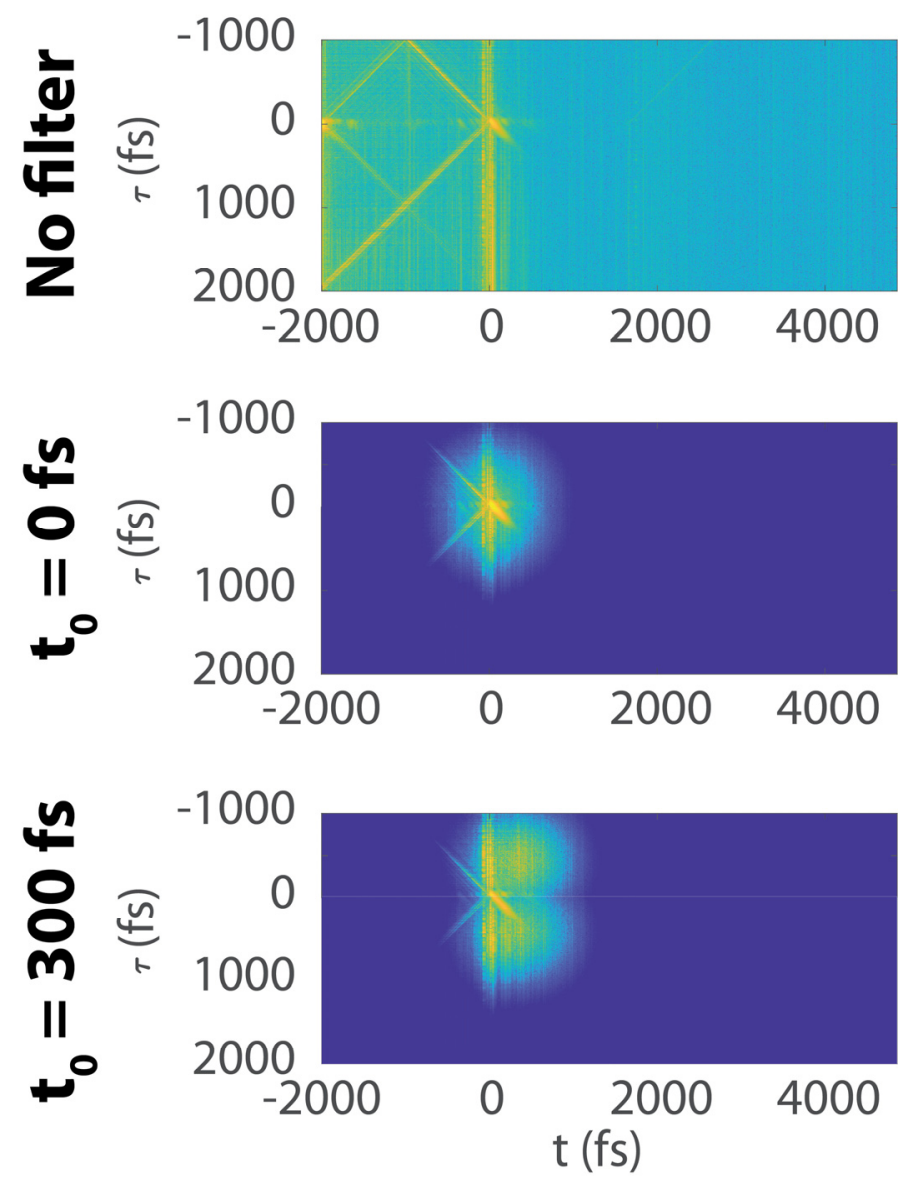

Figure S3. Representative ( $\tau, \mathrm{T}, t)$ domain plots are shown with no filter, and $t_{0}^{\prime}=0$ or $300 \mathrm{fs}$ at $T$ $=0 \mathrm{fs}$. The Lorentz-Gauss filters are applied along both the $\tau$ and $t$ domains. The arcsinh of the absolute values are shown in this figure to facilitate visual representation, because a linear mapping does not show the Lorentz-Gauss window function's contours well. (Note that all other figures have a linear color mapping.) The data are obtained by measurement. 
shown in fig. S4. Though the filter is applied in both $\tau$ and $t$ in the data analysis, this example that filters along $t$ only is illustrative of how increasing $t_{0}^{\prime}$ affects the experimental data. Alternative FMO spectra from HEOM, calculated with a site VIII wavenumber of either 12500 or $12700 \mathrm{~cm}^{-1}$, are shown in Figure S5. The peak position corresponding to site VIII appears near $12500 \mathrm{~cm}^{-1}$ or $12700 \mathrm{~cm}^{-1}$ depending on the site VIII wavenumber, showing that the filtered spectra are responsive to changes in the underlying Hamiltonian.
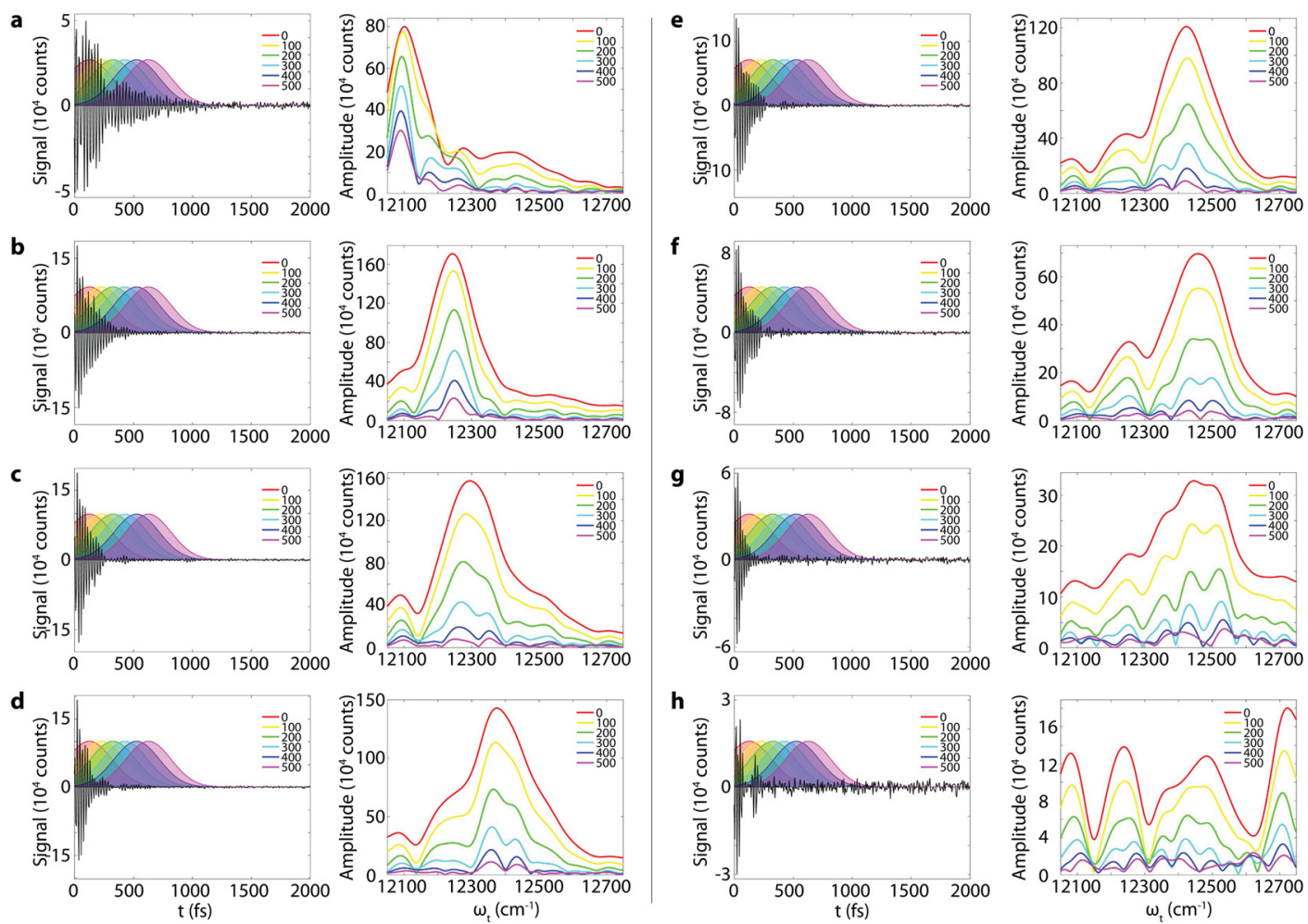

Figure S4. Line cuts taken from the rephasing data of a measured 2D spectrum at $T=60 \mathrm{fs}$ are listed at representative frequencies $\omega_{\tau}=12121,12274,12350,12415,12454,12520,12606$, and $12700 \mathrm{~cm}^{-1}$ (a-h, respectively). For each set of plots, the time- (left) and wavenumber-domain (right) line cuts are shown along the rephasing domain. The time-domain figures show LorentzGauss filters at different color-coded central positions $\left(t_{0}^{\prime}\right)$, which are multiplied by the data and subsequently Fourier transformed to yield the spectra shown in the corresponding color-coded wavenumber-domain spectra. At $t_{0}^{\prime}=0$, the peaks are broadened and less distinguishable within the broad peak shape. As $t_{0}^{\prime}$ increases the peaks narrow and become distinguishable; but the signal also decreases, placing an upper limit on $t_{0}^{\prime}$. 


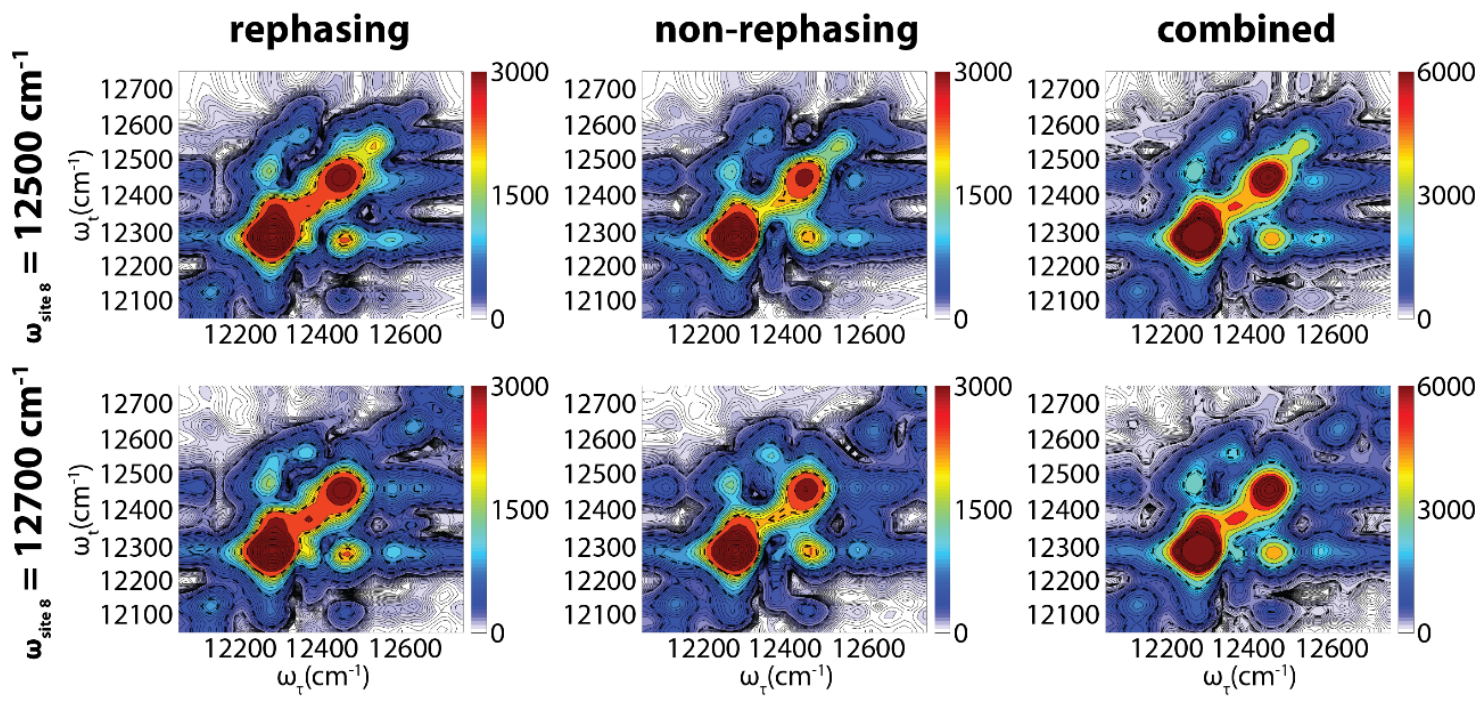

Figure S5. The rephasing, non-rephasing, and combined 2D spectra are shown, obtained using a Lorentz-Gauss filter with $t_{0}^{\prime}=300$ fs. These spectra were calculated using HEOM. The site VIII energy used to calculate the spectra was either 12500 or $12700 \mathrm{~cm}^{-1}$. The absolute values of the spectra are shown. 


\section{Rephasing and non-rephasing contributions in the FMO spectra}
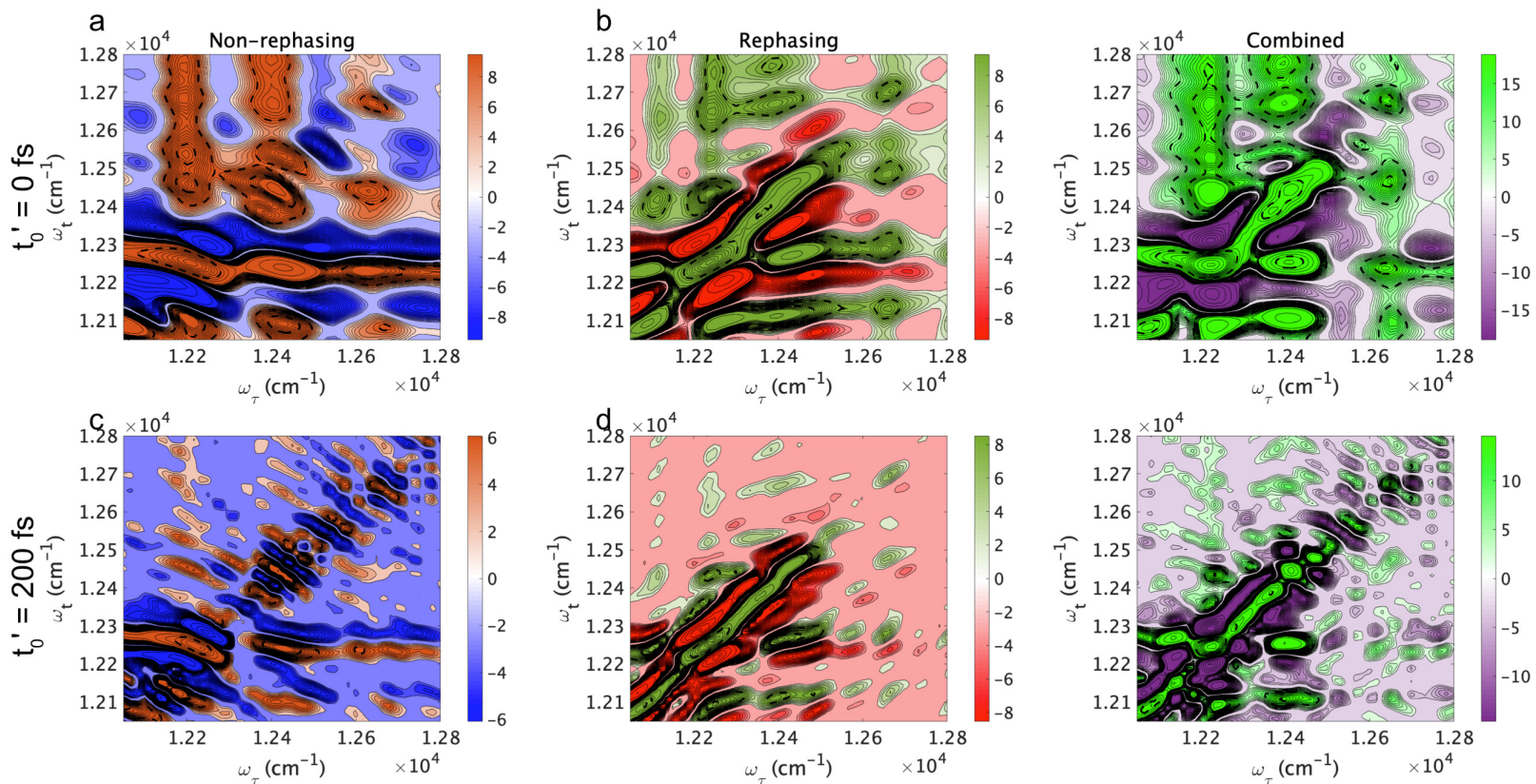

Figure S6. The 2D rephasing, non-rephasing, and combined spectra are shown at a representative timing $T=0 \mathrm{fs}$, applying the Lorentz-Gauss filter at $t_{0}^{\prime}=0$ (top) or $200 \mathrm{fs}$ (bottom). At $t_{0}^{\prime}=0$, the spectral features are broad and overlapping. In contrast, at $t_{0}^{\prime}=200 \mathrm{fs}$ the features are more distinguishable. The dotted lines indicate cutoffs where the contour step size has changed. The phased spectra are shown. These spectra were obtained by experimental measurement.

Figure S6 shows the non-rephasing, rephasing, and combined spectra obtained at $t_{0}^{\prime}$ $=0$ and $200 \mathrm{fs}$ from a single representative waiting time ( $T=0 \mathrm{fs})$. The individual peak shapes are distinguishable at individual waiting times. 


\section{The phasing and reproducibility of the 2D spectra}

Non-collinear 2DES and transient absorption (TA) both generate similar signals, however 2DES has an uncertain phase relation between its local oscillator and signal onset. Phasing is a method to recover this phase information, by comparing the 2DES and TA spectra. A thorough description was published by Brixner et al.,7 but a brief background will be presented here.

2DES and TA both generate signals via a third-order hyperpolarization $P^{(3)}(t)$ (equation S9).

$P^{(3)}(t)=\int_{0}^{\infty} \int_{0}^{\infty} \int_{0}^{\infty} S^{(3)}\left(t_{1}, t_{2}, t_{3}\right) E\left(t-t_{1}-t_{2}-t_{3}\right) E\left(t-t_{2}-t_{3}\right) E\left(t-t_{3}\right) d \tau d T d t$

This hyperpolarization depends on the sample's third-order response function $S^{(3)}\left(t_{1}, t_{2}, t_{3}\right)$ and the electric fields $E(\omega, t)=A \exp (i \omega t-i \vec{k} \cdot \vec{r})$ involved in each of three interactions between the sample and light, where $A=E_{0} \vec{\varepsilon} \exp (-i \varnothing)$ is the complex pulse envelope, $E_{0}$ is the electric field amplitude, $\vec{\varepsilon}$ is the field's unit vector, $\omega$ is frequency, $k$ is wavenumber, $r$ is position, $\varnothing$ is a phase offset, $t$ is time, and $t_{n}$ is the timing of the $n$th interaction with light.

The sample characteristics contributing to the signal are therefore described by $S^{(3)}\left(t_{1}, t_{2}, t_{3}\right)$, while the intensity of the signal is also dependent on the timing, phases, and amplitudes of the three electric fields. The interactions can happen any time within the temporal envelope of the incident pulses, so the timing cannot be controlled exactly. However, their average timing can be controlled by the timing of the incident pulses. Furthermore, because the spectra of all incident pulses are identical in this study, the signal scales with the intensity of the incident light as $P^{(3)}(\omega) \propto I(\omega)^{3 / 2}$. 
In order to compare the 2DES and TA signals, the projection-slice theorem is invoked to reduce the 2DES signal to one dimension by integrating over its $\omega_{\tau}$ axis at a particular waiting time to yield a spectrum along the $\omega_{t}$ domain. The equation we use is shown below (equation S10). Here, we assume the index of refraction to be 1 .

$A\left(T, \omega_{t}\right)=\operatorname{Re}\left[\omega_{t} \int_{-\infty}^{\infty} S^{(3)}\left(\omega_{\tau}, T, \omega_{t}\right) \exp \left(i\left[\emptyset_{1}+\emptyset_{2} t+\emptyset_{3} t^{2}\right]\right) d \omega_{\tau}\right]$

Here, $\emptyset_{i}$ are phase correction coefficients expanded in a Taylor series with respect to $t$, and $\omega_{0}$ is the light frequency. The phase terms are fit to the TA signal. Because the total signal intensity is proportional to the product of the system response and the probe beam's electric field contributions, ${ }^{7}$ this TA signal is divided by the square-root of the probe beam intensity before comparison to the 2DES signal.

The phased spectra from 2DES are shown alongside the corresponding TA spectrum in Figure S7. Here, the 2DES spectra are obtained by using a $400 \mathrm{fs}$ apodization window in the coherence time domain and $600 \mathrm{fs}$ in the rephasing-time domain following the arrival of beam 3. Hanning windows were applied to the spectra to reduce the artifacts from sharp cut-offs in the corresponding frequency domain due to Fourier transform. 

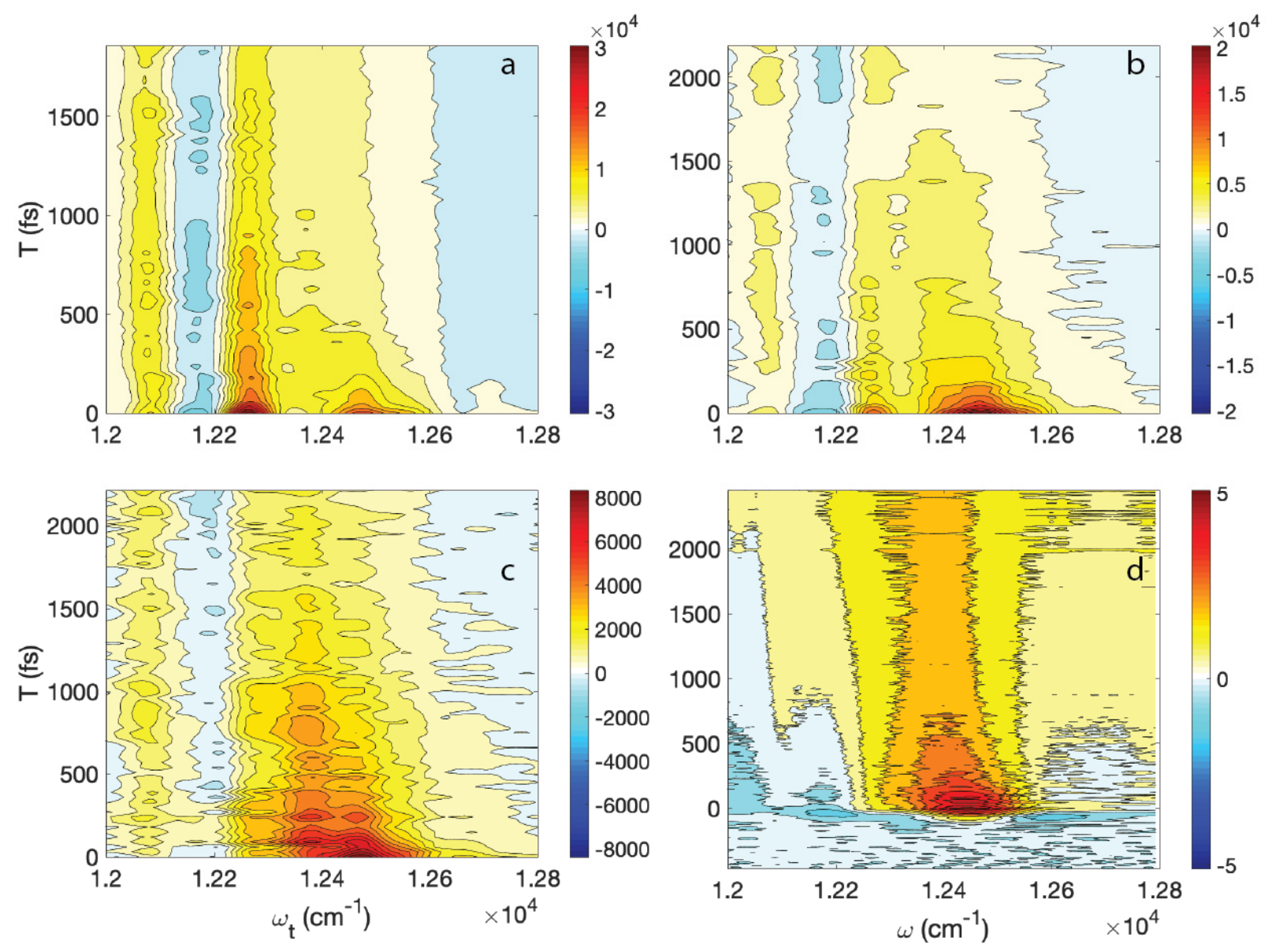

Figure S7. Phased spectra obtained from 2DES measurements on Days 1-3 (a-c, respectively) are shown, compared to a measured TA spectrum (d). The spectra from 2DES are obtained using equation S10. The TA spectrum is obtained by subtracting the probe signal from the pump+probe signal, and dividing by the square-root of the probe signal.

The TA spectra contain several features. A dominant, positive signal contribution appears from approximately $12,300-12,600 \mathrm{~cm}^{-1}$. The blue side of this signal partially decays within approximately $300 \mathrm{fs}$, leading to a dominant feature at approximately 12,400 $-12,500 \mathrm{~cm}^{-1}$. The region spanning $12,000-12,250 \mathrm{~cm}^{-1}$ contains overlapping positive and negative contributions. The positive feature appears near $12,100 \mathrm{~cm}^{-1}$, while negative features appear at either side. 
In comparison, the 2DES signals contain several similar features, as well as some differences that will be addressed subsequently. Near zero waiting time, they all contain a dominant signal contribution in the region of $12,400-12,600 \mathrm{~cm}^{-1}$. The blue side of this peak has a quickly decaying component, and then a slower-decaying peak centered near $12,500 \mathrm{~cm}^{-1}$. A negative feature appears at approximately $12,200 \mathrm{~cm}^{-1}$, and then a positive peak at approximately $12,100 \mathrm{~cm}^{-1}$. They all contain a relatively weak signal component near zero waiting time in the region from $12,600-12,800 \mathrm{~cm}^{-1}$. They also contain the following differences. First, the negative signal components persist at $12,200 \mathrm{~cm}^{-1}$, instead of gradually lifting to a positive signal as in the TA spectrum.

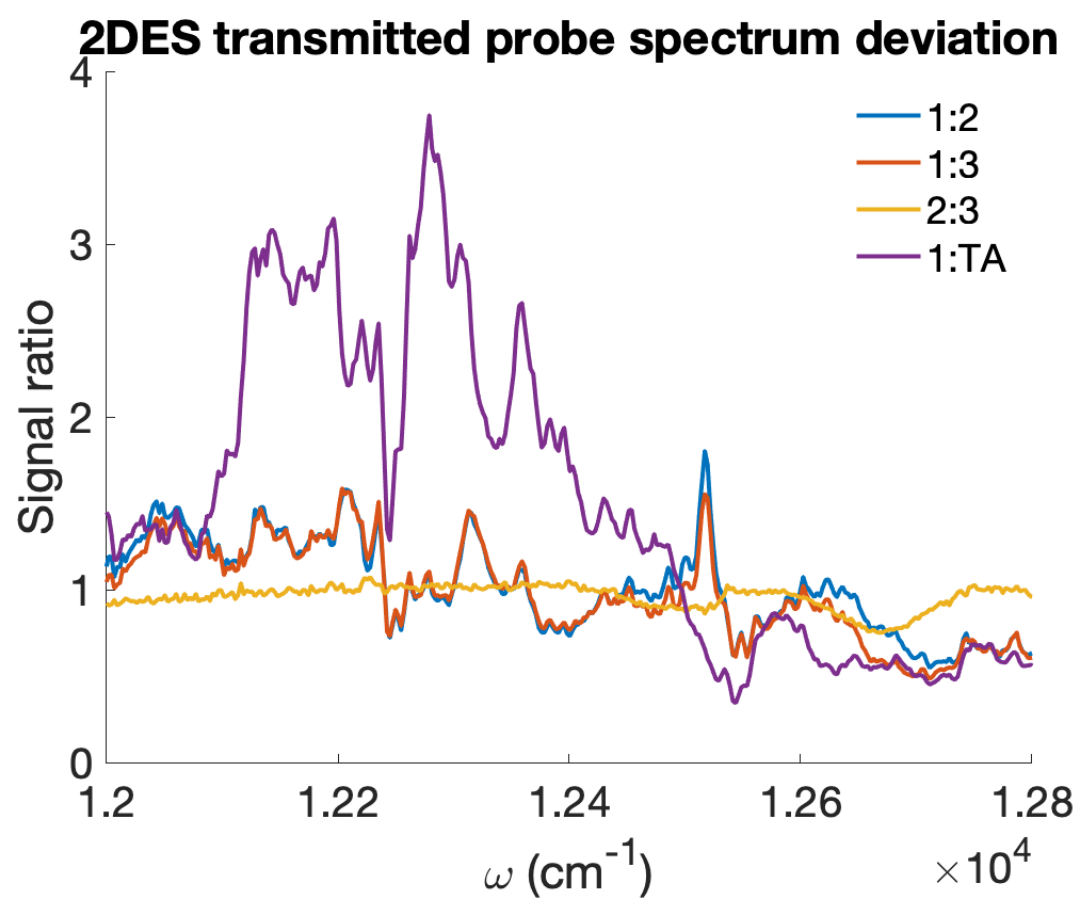

Figure S8. The intensity ratios of the transmitted probe spectra are shown from Days 1-3 and the signal from the TA spectrum. Day 1 deviated the most from the others, because the experimental apparatus was tuned between experiments from Day 1 and Day 2. Data from Days 2-3 were measured in succession, without re-tuning, resulting in more similar incident laser spectra. 
Furthermore, the peak at $12,300 \mathrm{~cm}^{-1}$ is especially intense in the data from Day 1 , compared to those from Days 2-3. The regions at $12,400 \mathrm{~cm}^{-1}$ and $12,600 \mathrm{~cm}^{-1}$ are weaker in Day 1 than Days 2-3, while the region at $12,500 \mathrm{~cm}^{-1}$ is stronger, leading to a similar signal shape in the transient spectra with a sharper peak at $12,500 \mathrm{~cm}^{-1}$ and weaker shoulders on either side of this peak compared to the Day 2 and 3 spectra. The 12,300 $\mathrm{cm}^{-1}$ peak in the Day 2 data also has more relative intensity compared to that from Day 3, as well as diminished intensity at approximately $12,350 \mathrm{~cm}^{-1}$. Though this feature is the smallest in Day 3, it nonetheless is still evident as a shoulder overlapping with its higher-energy peaks. As the $12,300 \mathrm{~cm}^{-1}$ peak becomes more intense, it out-competes the intensity from the peaks at $12,400-12,600 \mathrm{~cm}^{-1}$, so that this bluer region becomes diminished by comparison on the color maps of Days 1 and 2. To explain this peak's prominence on Day 1, the ratios of the transmitted probe spectra obtained on 2DES experiments from Days 1-3 are shown in Figure S8. This figure shows the ratios of the transmitted probe spectra obtained on Days 1-3. On Day 1, there is approximately $50 \%$ more intensity in the incident beams at 12,300 $\mathrm{cm}^{-1}$ compared to the 2D signal on other days, accounting for the extra intensity in this region. Meanwhile, the relative strength of the $12,300 \mathrm{~cm}^{-1}$ peak in the Day 2 data can be accounted for by the diminished intensity near $12,500 \mathrm{~cm}^{-1}$ which, relative to the Day 3 data, reduces the intensity of this feature compared to the peak at $12,300 \mathrm{~cm}^{-1}$.

2D and transient absorption spectra at particular waiting times are shown in Figure S9. The peaks at $<12300 \mathrm{~cm}^{-1}$ are heightened in the 2D spectra, coinciding with increased incident light in that region compared to the TA spectrum (Figure S8). The TA spectrum was measured using the same spectrum for its pump and probe beams. The spectral line shape in the rest of the spectra offer a good match consistently in the remaining parts of the spectra, 
for the duration of the scan. Like the transient absorption spectrum in Figure S7d, the exciton 1 peak at $12102 \mathrm{~cm}^{-1}$ grows as $\mathrm{T}$ increases compared to the rest of the spectral peaks, indicating downhill energy transfer.
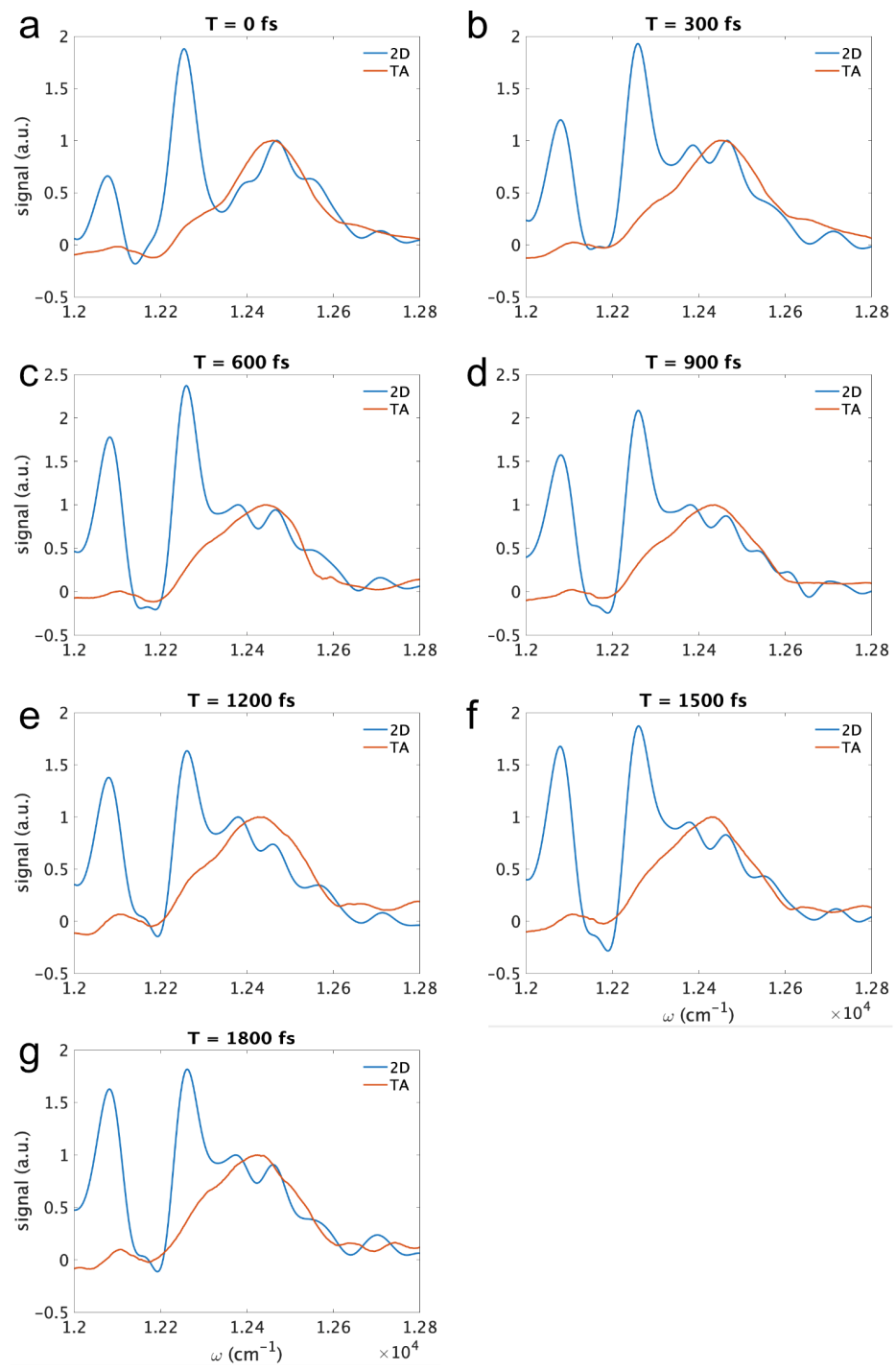

Figure S9. Phased 2D spectra (after summation along the $\omega_{\tau}$ domain) are plotted with the transient absorption (TA) spectra at the same time delay, in the waiting time range of 0 $1800 \mathrm{fs}$ with time steps of $300 \mathrm{fs}$ (a-g, respectively). As described in Figure S8 and the accompanying text, the higher beam power at $<12200 \mathrm{~cm}^{-1}$ and at $12300 \mathrm{~cm}^{-1}$ lead to increased peak intensities at these positions in the 2D spectrum, compared to the TA spectra. However, the remaining 2D peaks coincide with the positions in the TA spectra. 


\section{Aliasing in the experimental and computed line shapes}

The computed spectra were generated using a step size of 1 fs (Figure S10). In contrast, the experimental measurements were performed with a 3.5 fs step size, introducing an aliasing effect. Aliasing occurs in 2D spectra when the time-domain step size exceeds half the signal's optical cycle. In this case, $800 \mathrm{~nm}$ light has an optical period of 2.67 fs, so aliasing occurs if the $\tau$-domain step size surpasses approximately $1.33 \mathrm{fs}$. Here, we test whether the aliasing significantly affects the signal output using computed spectra. Because the computations were performed with a step size of $1 \mathrm{fs}$, they do not feature aliasing. However, by selecting every third data point in the data set, we obtain an aliased example with a step size of 3 fs (Figure S11).

If aliasing significantly distorts the spectra, it will be apparent by comparing the spectra obtained by these two cases shown in Figs. S10 and S11. To simplify the comparison, we select the line cut of the exciton 2 signal in the $\omega_{t}$ domain due to its large intensity. The $\omega_{\tau}$-domain signals in these unaliased and aliased cases are shown in Figure S12. Because the number of data points in each case is odd ( $N=2049$ points), the frequency domains were obtained by using Nyquist frequencies of $\pm \frac{1}{2 \Delta \tau}$ as the outer limits, and then spacing $\mathrm{N}$ data points with constant step size between these two limits, where $\Delta \tau$ is the step size. We also did likewise for $\Delta t$ in the rephasing-time domain. Because the aliased example has a step size of $3 \mathrm{fs}$, which is slightly more than two times $1.33 \mathrm{fs}$, the aliased frequency axis was shifted by adding two times $\frac{1}{2 \Delta \tau}$ to restore to the frequency positioning lost by aliasing. These spectra have very similar line shapes, with an apparent peak shift of 
$5 \mathrm{~cm}^{-1}$. The aliased signal has a slightly raised line shape on either side of the peak. Overall, the differences are minor.
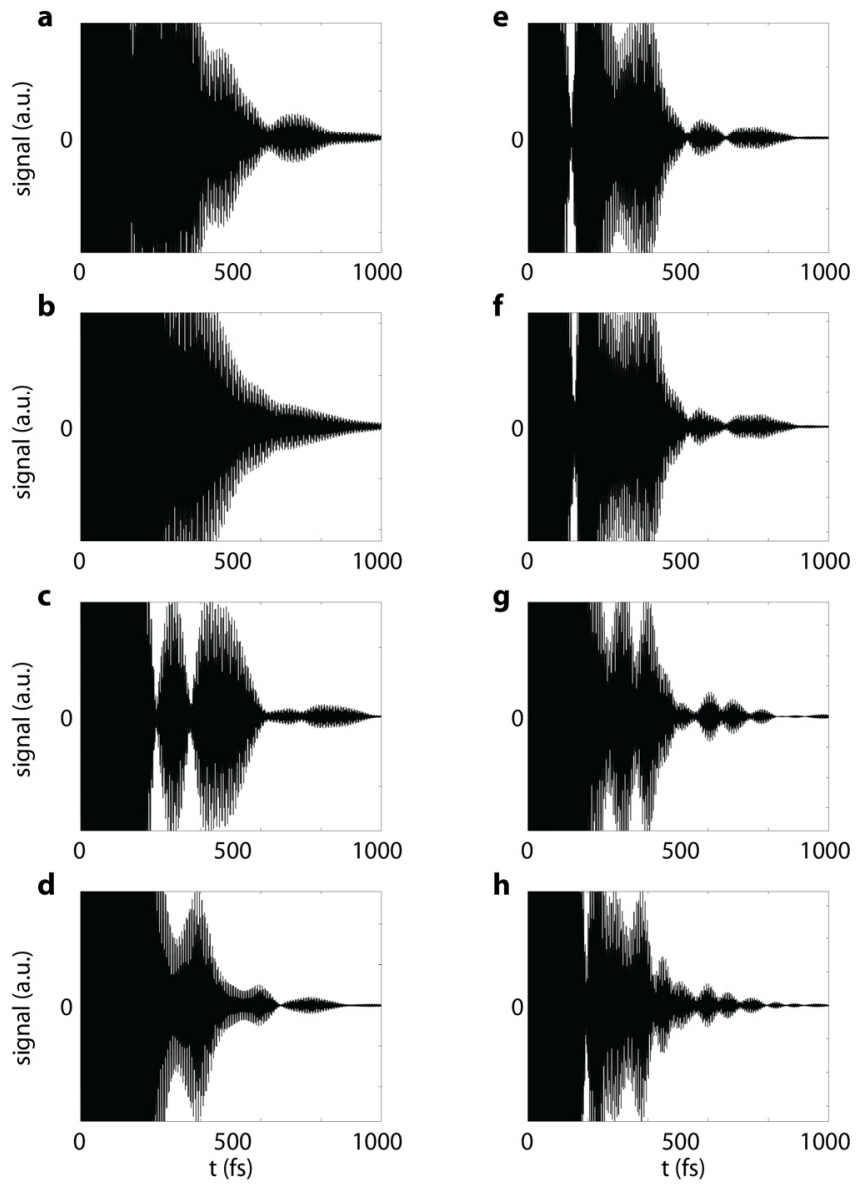

Figure S10. Time-domain calculated line shapes are shown at $\omega_{t}=12133,12268,12360$, $12435,12495,12518,12618$, and $12740 \mathrm{~cm}^{-1}(\mathrm{a}-\mathrm{h})$. The step size in the $\tau$-domain is $1 \mathrm{fs}$. 

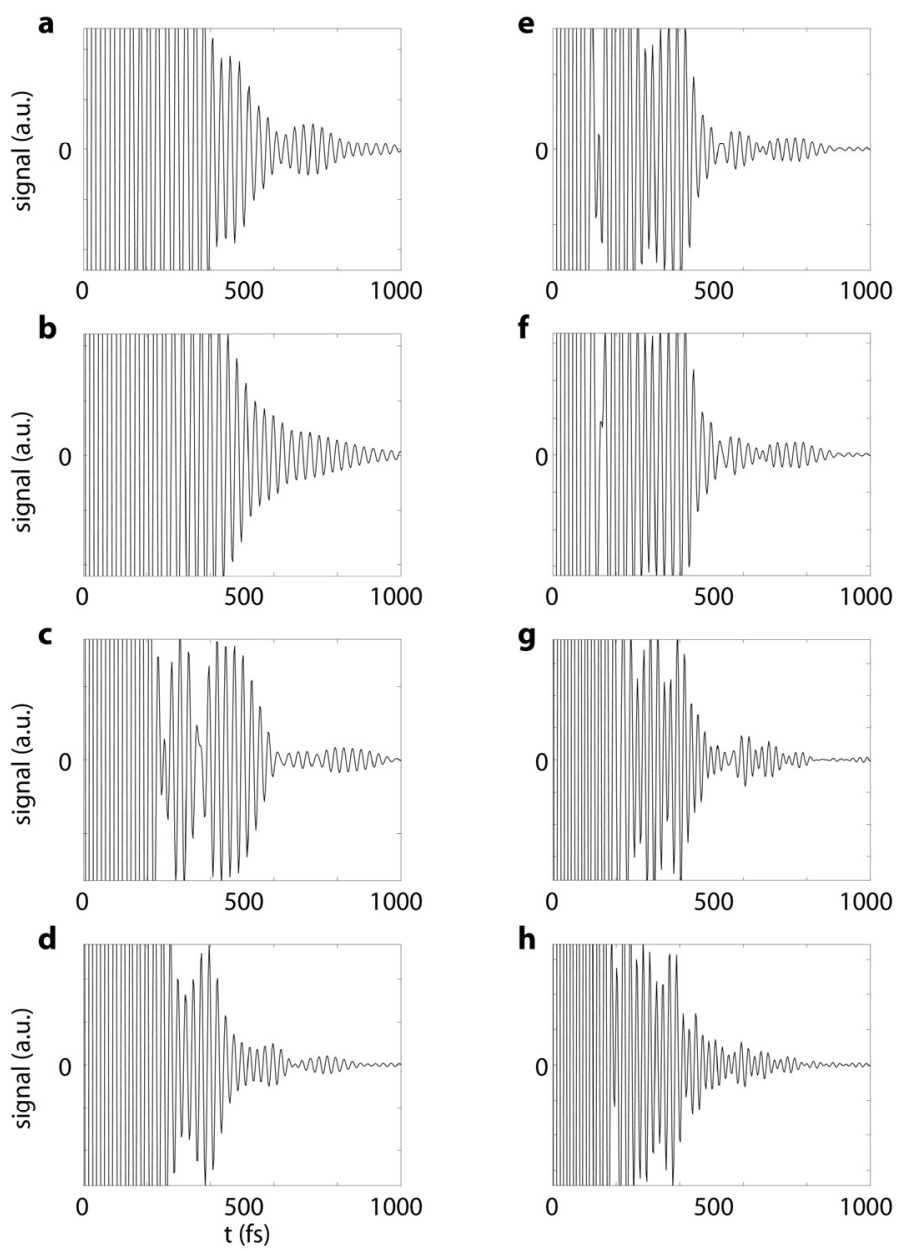

Figure S11. Time-domain calculated line shapes are shown at $\omega_{\tau}=12133,12268,12360$, $12435,12495,12518,12618$, and $12740 \mathrm{~cm}^{-1}(\mathrm{a}-\mathrm{h})$. The calculations are shown with a $3 \mathrm{fs}$ step size, obtained by collecting every third data point compared to the data shown in Figure S9. 


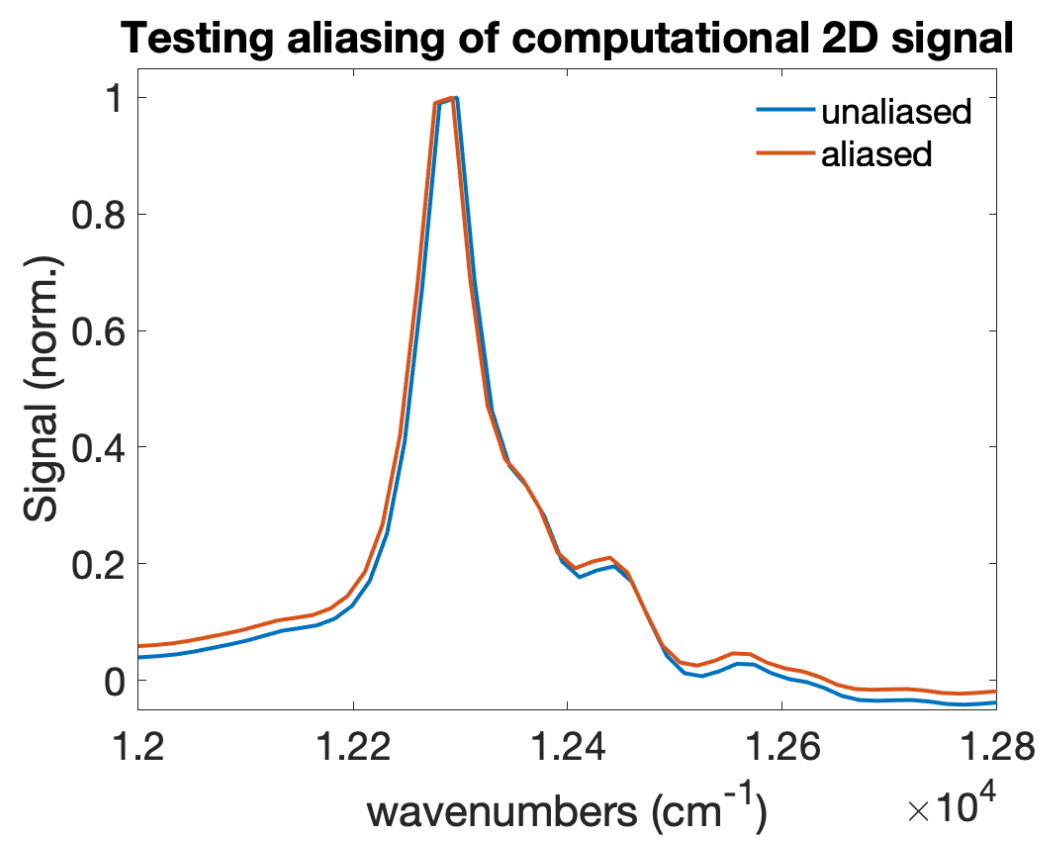

Figure S12. Spectral components of the computed FMO spectrum are shown for $\tau$ and $t$ step sizes of $1 \mathrm{fs}$ (blue) and $3 \mathrm{fs}$ (red), corresponding to the unaliased and aliased cases, respectively. The position and line shape are not significantly changed by the difference in the sampling period. The signal used here corresponds to exciton 2 in the $\omega_{t}$ domain. Because this is a line cut from the 2D spectrum, this spectrum contains cross-peaks with the $\omega_{\tau}$ domain, due to other excitons embedded in its shoulders. 


\section{The effect of random noise and vibrational contributions}

To simulate the random noise contribution, we start with the data corresponding to the HEOM-simulated 2D spectra (Fig. S10). We add random noise $N_{\mathrm{j}}$ to each data point $j$, according to equation S11.

$$
N_{j}=M F r_{j}
$$

In this equation, $M$ is the maximum of the real-valued time-domain data; $F$ is a scalar multiple that tunes the amount of noise added (indicated in the legend of Figure S13); and $r_{j}$ is a random number (with a flat probability density) in the range of $[-0.5,0.5]$.
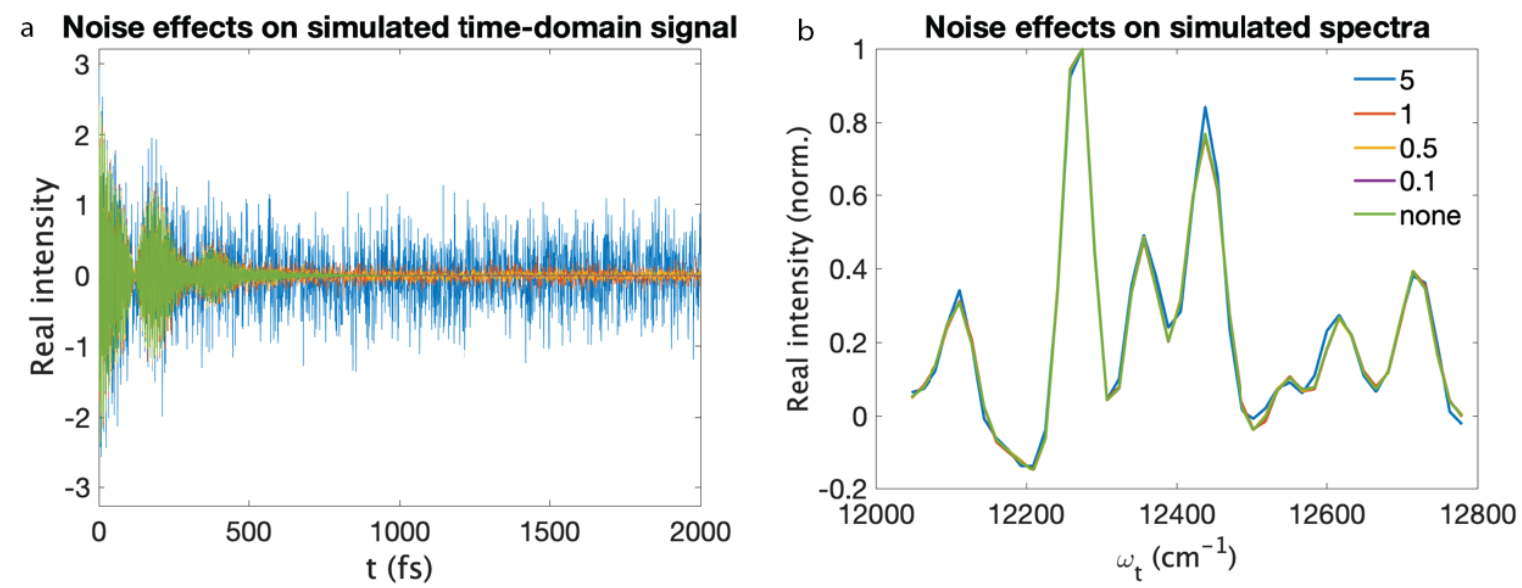

Figure S13. Time-domain (a) and wavenumber-domain (b) signals are shown, calculated from HEOM with added noise. The legend indicates the noise factor $F$, according to equation S8. These plots are generated by summing 63 independent instances of noisy 2D spectra to simulate the 63 steps in the T-domain of our experimental data. Each spectrum was generated by adding noise point-by-point to the time-domain of the 2D spectrum shown in Figure S9, then summing over the coherence frequency and waiting time domains. This is the same methodology that generated the spectra in Fig. 5 of the main text. Part (b) shows that the noise does not significantly alter the lineshape in the corresponding wavenumber-domain spectra.

Here, we sum over the T-domain steps in the main text. Figure S13 shows the real-valued time- and wavenumber-domain data resulting from the addition of this noise, after performing the same summations used for the experimental data in Figure 4 in the main text. We assigned values as high as 5 to $F$, which is a significant overestimate of the noise we would 
observe in actual experiments. From these spectra, we observe that noise does not contribute significantly to the spectra in the wavenumber window of interest.

For the vibrational sublevel contributions, we performed an HEOM calculation to simulate the 2D signal for sites 3 and 4 of FMO as a representative example. The reason for this calculation is that it is too computationally expensive to calculate the spectrum of the entire 8-site system if one additional vibrational sublevel is included on each existing state.

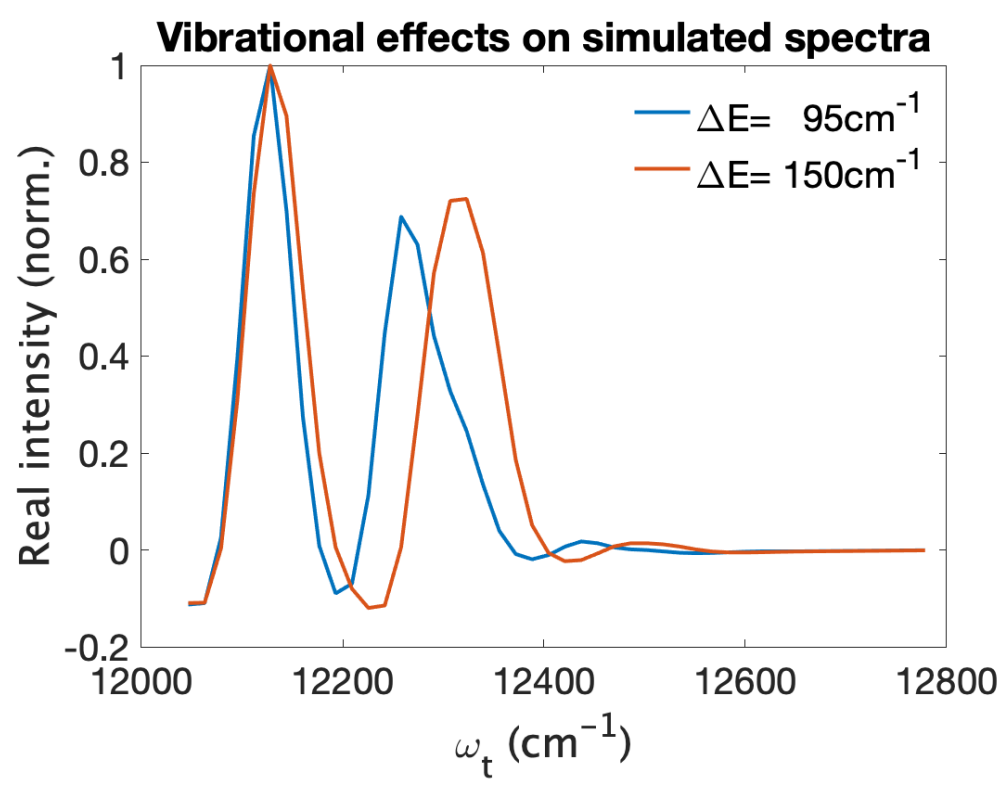

Figure S14. 2D spectra were calculated using HEOM, for a system that includes two chromophores representing sites 3-4 of FMO. One additional vibrational sublevel was included for each state. The spectra were summed over the $\omega_{\tau}$ domain, yielding the $\omega_{t}$-domain spectra shown here, similar to those shown in Figs. 1e and 4 of the main text. The energy gap of the electronic states was tuned closer to (red) or further from (blue) the vibrational energy spacing. Coincidence of these energy gaps would result in electronic-vibrational mixing, allowing the vibrational modes to borrow maximal oscillator strength from the electronic states and producing a worst-case scenario for the appearance of vibrational overtone features in the filtered spectra. However, no such peaks appear when comparing the two cases.

The following parameters were used: $\mathrm{E}_{1}=12150 \mathrm{~cm}^{-1}$ and $\mathrm{E}_{2}=12245$ (blue) or $12305 \mathrm{~cm}^{-1}$ (red) for the two chromophores' excited states (figure S14); and V $=-50 \mathrm{~cm}^{-1}$ coupling. These parameters coincide with poorly mixed and well-mixed electronic-vibrational states, 
respectively (Figure S15). The vibrational mode is set to $180 \mathrm{~cm}^{-1}$ with a Huang-Rhys factor of 0.025 . The temperature is $77 \mathrm{~K}$, with a bath relaxation time of $100 \mathrm{fs}$ and reorganization energy of $35 \mathrm{~cm}^{-1}$. The parameterization involving a $150 \mathrm{~cm}^{-1}$ electronic energy offset is based on a previous publication, ${ }^{8}$ though with a global energy shift.

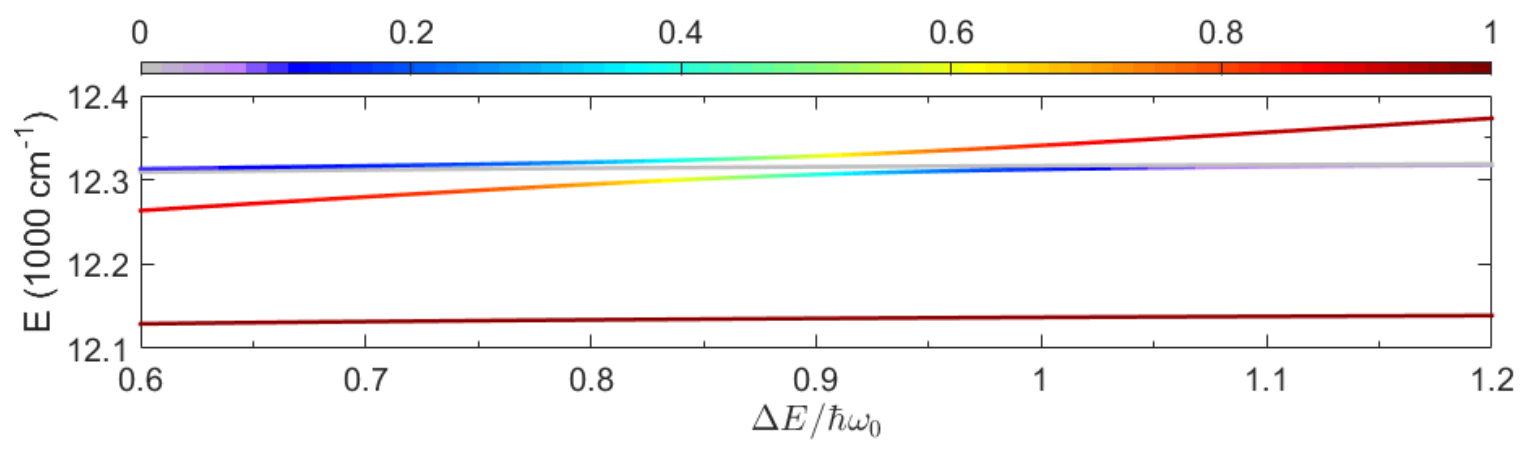

Figure S15. The energies and oscillator strengths of the excited states are shown, calculated as a function of the electronic energy gap $\Delta E$ compared to the vibrational energy spacing $\hbar \omega_{0}$. The mixing between electronic and vibrational states is most significant when $\Delta E / \hbar \omega_{0}$ is approximately 0.88 . The color scale indicates the oscillator strength.

In this simulated spectrum, we have considered two energy configurations. In one case, the vibrational level is not similar to the energy gap of the electronic excited states, which minimizes their mixing and serves as a "control" test. In the other case, the vibrational level is similar to this energy gap, which induces quantum mixing between the electronic and vibrational states and maximizes the possibility of seeing an effect in the spectrum, due to the vibrational signals' "borrowing" oscillator strength from the electronic transitions. When comparing these cases, we do not see any new peaks arise in the latter case. Therefore, we see no discernable effects arising from vibronic character using known parameters for our system.

\section{Comparison to previously published $2 \mathrm{D}$ spectra}


The line shapes of 2D spectra depend on the sample preparation,experimental, and analysis conditionslaser spectrum. For example, Sec. 6 discusses that 2D spectra depend on the pump beams' spectral intensity by $P^{(3)}(\omega) \propto I(\omega)^{3 / 2}$, and it describes how these characteristics impact the spectra presented here. Additionally, Orf et. al. ${ }^{9}$ reported a redox-statedependent photoprotection mechanism, and Allodi et al. ${ }^{10}$ reported how it can change the 2D spectra (eg., Figure 2 in that publication). Furthermore, the apparent resolution is also affected by the coherence-time range and windowing parameters, up to the signal duration. The spectra published by Engel et al. ${ }^{11}$ and Panitchayankoon et al. ${ }^{12}$ were treated with ascorbate, a reducing agent. Therefore, those spectra must be compared to the reduced sample's spectra in the publication by Allodi et al. ${ }^{10}$ which were reduced with dithionite, a stronger reducing agent. While we did not use ascorbate,a reducing agent, we minimized atmospheric exposure as best we could and our spectra nonetheless-resemble the reduced spectra-as well. Allodi et al. ${ }^{10}$ and Orf et $\mathrm{al}^{9}$ attribute the spectral differences in oxidized and reduced conditions primarily to changes in the local electrostatic environments of excitons 4,2 , and 1 , as well as changes to the system-bath coupling. The reduced condition is most relevant to physiological conditions for this anaerobic bacterium.

The spectra shown in the main text are summed over waiting time, but these other publications show the spectra at individual waiting times. Therefore, in Figure S16 we show the spectrum at $\mathrm{T}=30 \mathrm{fs}$, which is our closest example to the $\mathrm{T}=40 \mathrm{fs}$ spectrum showed by Allodi et al. For comparison to previously published spectra, we have applied smaller apodization window widths than $1 \mathrm{ps}$, more consistent with previous analyses $\left(\tau_{\max }=\right.$ $400 \mathrm{fs}$ and $t_{\max }=600 \mathrm{fs}$ ). Therefore, aside from the treatment of waiting time, the spectrum shown here also differs from the "unfiltered" spectrum shown in Fig. 3 of the main text, 
where the coherence and rephasing domain signals are preserved up to $1 \mathrm{ps}$ for the eventual application of the LG filter. One significant difference between the oxidized and reduced spectra, according to Figure 2 in the publication by Allodi et al., is the presence of positive signal at the $\left[\omega_{\tau}, \omega_{t}\right]$ coordinates of approximately $\left[12200-13000 \mathrm{~cm}^{-1}, 12650-13000 \mathrm{~cm}^{-1}\right]$ in the reduced case, but not in the oxidized case $\left(13000 \mathrm{~cm}^{-1}\right.$ is the approximate upper limit of their plot range). This feature is outside of the plot ranges for the publications by Panitchayankoon et al. and Engel et al. We observe positive signals in this spectral region. Elsewhere, the other publications find a negative band at approximately $\omega_{t}=12500 \mathrm{~cm}^{-1}$, which corresponds to a horizontal region where the signal strength decreases in Figure S16, however we do not resolve a negative signal here. However, in higher resolution spectra (Fig. 3 , main text) we observe the signal go to zero at this region, with slight negative peaks, for instance, at $\left[12400 \mathrm{~cm}^{-1}, 12500 \mathrm{~cm}^{-1}\right]$. The previous publications disagree about whether the signal is positive or negative in the region of $\left[12600-12700 \mathrm{~cm}^{-1}, 12600-12700 \mathrm{~cm}^{-1}\right]$, with Allodi et al. reporting negative signal there, while the other two report positive signal. We observe a positive peak at $\left[12700 \mathrm{~cm}^{-1}, 12700 \mathrm{~cm}^{-1}\right]$, but we also find a negative region directly underneath it. Like these other publications, we observe similar positive features at approximately $\omega_{t}=12100 \mathrm{~cm}^{-1}$ and $12270 \mathrm{~cm}^{-1}$ that spans most of the $\omega_{\tau}$ range, a negative feature above the diagonal line at $\left[12100 \mathrm{~cm}^{-1}, 12200 \mathrm{~cm}^{-1}\right]$, and another at $\left[12200 \mathrm{~cm}^{-1}\right.$, $\left.12350 \mathrm{~cm}^{-1}\right]$.

We also observe some differences among the spectra. For example, the spectra in Panitchayankoon et al. and those at $\mathrm{T}>0$ fs by Engel et al. do not contain the negative signal at $\omega_{t}=12050 \mathrm{~cm}^{-1}$ shown in the other reports. This negative signal is not resolved in Fig. S16, however it is observable when longer time delays are included (Fig. 3, main text). 
Panitchayankoon et al. and Engel et al. do not report a clear separation between the peak structures near $\left[12250 \mathrm{~cm}^{-1}, 12100 \mathrm{~cm}^{-1}\right]$, though Allodi et al. do see it. We observe signs of it in Fig. S16, but it is much better resolved in Fig. 3 of the main text. These distinctions may be due to the apparent spectral resolution, as well as differences in the incident spectra. For the reasons stated in the beginning of this section, we would not expect exact coincidence of the spectra. Nonetheless, many of the features are qualitatively similar.

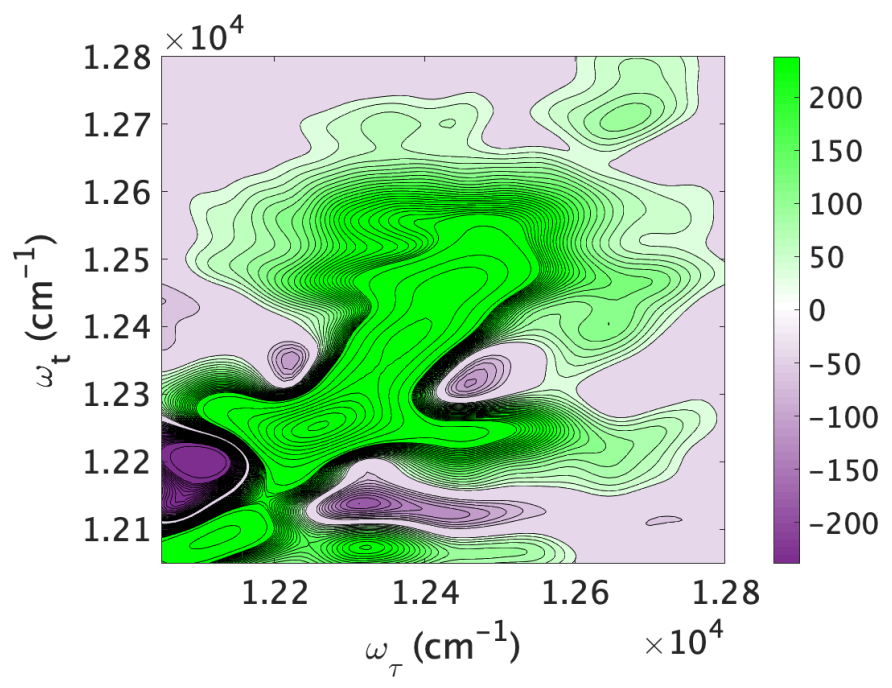

Figure S16. The 2D spectrum of the combined rephasing and non-rephasing measurements is shown at $\mathrm{T}=30$ without $\mathrm{LG}$ filtering. This spectrum was chosen because it is the closest to the spectra at 40 fs shown by Allodi et al. ${ }^{10}$ To compare to previous spectra, for the Hanning apodization windows we have used the coherence and rephasing time domain maxima of $\tau_{\max }=400 \mathrm{fs}$ and $t_{\max }=600 \mathrm{fs}$.

\section{Supplemental references}

1. Zheng, H.; Caram, J. R.; Dahlberg, P. D.; Rolczynski, B. S.; Viswanathan, S.; Dolzhnikov, D. S.; Khadivi, A.; Talapin, D. V.; Engel, G. S., Dispersion-free continuum two-dimensional electronic spectrometer. Appl. Opt. 2014, 53 (9), 1909-1917.

2. Tanimura, Y.; Kubo, R., Time Evolution of a Quantum System in Contact with a Nearly Gaussian-Markoffian Noise Bath. J. Phys. Soc. of Japan 1989, 58, 101-114. 
3. Feynman, R. P.; Vernon Jr, F. L., The Theory of a General Quantum System Interacting with a Linear Dissipative System. Annals of Physics 2000, 281, 547-607.

4. Yeh, S.-H.; Kais, S., Simulated two-dimensional electronic spectroscopy of the eightbacteriochlorophyll FMO complex. J. Chem. Phys. 2014, 141 (23), 234105.

5. Shi, Q.; Chen, L.; Nan, G.; Xu, R.-X.; Yan, Y., Efficient hierarchical Liouville space propagator to quantum dissipative dynamics. J. Chem. Phys. 2009, 130 (8), 084105-5.

6. (a) Chen, L.; Zheng, R.; Jing, Y.; Shi, Q., Simulation of the two-dimensional electronic spectra of the Fenna-Matthews-Olson complex using the hierarchical equations of motion method. j. Chem. Phys. 2011, 134 (19), 194508; (b) Hein, B.; Kreisbeck, C.; Kramer, T.; Rodríguez, M., Modelling of oscillations in two-dimensional echo-spectra of the Fenna-Matthews-Olson complex. New J. Phys. 2012, 14 (2), 023018.

7. Brixner, T.; Mančal, T. s.; Stiopkin, I. V.; Fleming, G. R., Phase-stabilized twodimensional electronic spectroscopy. J. Chem. Phys. 2004, 121 (9), 4221-4236.

8. Fujihashi, Y.; Fleming, G. R.; Ishizaki, A., Impact of environmentally induced fluctuations on quantum mechanically mixed electronic and vibrational pigment states in photosynthetic energy transfer and 2D electronic spectra. j. Chem. Phys. 2015, 142 (21), 212403.

9. $\quad$ Orf, G. S.; Saer, R. G.; Niedzwiedzki, D. M.; Zhang, H.; McIntosh, C. L.; Schultz, J. W.; Mirica, L. M.; Blankenship, R. E., Evidence for a cysteine-mediated mechanism of excitation energy regulation in a photosynthetic antenna complex. Proc. Natl. Acad. Sci. USA 2016, 113 (31), E4486-E4493.

10. Allodi, M. A.; Otto, J. P.; Sohail, S. H.; Saer, R. G.; Wood, R. E.; Rolczynski, B. S.; Massey, S. C.; Ting, P.-C.; Blankenship, R. E.; Engel, G. S., Redox Conditions Affect Ultrafast Exciton Transport in Photosynthetic Pigment-Protein Complexes. J. Phys. Chem. Lett. 2018, 9, 89-95.

11. Engel, G. S.; Calhoun, T. R.; Read, E. L.; Ahn, T.-K.; Mančal, T. s.; Cheng, Y.-C.; Blankenship, R. E.; Fleming, G. R., Evidence for wavelike energy transfer through quantum coherence in photosynthetic systems. Nature 2007, 446 (7137), 782-786.

12. Panitchayangkoon, G.; Hayes, D.; Fransted, K. A.; Caram, J. R.; Harel, E.; Wen, J.; Blankenship, R. E.; Engel, G. S., Long-lived quantum coherence in photosynthetic complexes at physiological temperature. Proc. Nat. Acad. Sci. USA 2010, 107 (29), 12766-12770. 